\title{
A cost effective non-commercial ECL-solution for Western blot detections yielding strong signals and low background
}

\author{
Claude Haan*, Iris Behrmann \\ Laboratoire de Biologie et Physiologie Intégrée, Fac. Science Techno. \& Com., Université du Luxembourg, \\ 162a, avenue de la Faïencerie, L-1511 Luxembourg, Luxembourg
}

Received 7 April 2006; received in revised form 13 July 2006; accepted 14 July 2006

Available online 2 November 2006

\begin{abstract}
We compared several alternative ECL solutions for Western blot detection of endogenous proteins in whole cell lysates using inexpensive, commercially available reagents. Starting from an existing protocol based on $p$-coumaric acid (pCA) as enhancer, we found that the ECL solution containing 4-iodophenylboronic acid (4IPBA) generated strong specific signals and low background chemiluminescence. We optimised the luminol, 4IPBA and hydrogenperoxide concentrations of this 4IPBA-ECL solution. The optimised 4IPBA-ECL solution (100 mM Tris/HCl pH 8.8, $1.25 \mathrm{mM}$ luminol, $2 \mathrm{mM}$ 4IPBA, $5.3 \mathrm{mM}$ hydrogenperoxide) shows a greatly increased signal intensity compared to the initial pCA-ECL protocol and to some commercially available ECL solutions. In addition, the optimised 4IPBA-ECL solution also generates much lower background chemiluminescence than other noncommercial ECL solutions using $p$-coumaric acid or 4-iodophenol as enhancers. The 4IPBA-ECL solution was stable when stored but had the lowest background when prepared freshly from stock solutions. Thus, we present an optimised protocol for a wellperforming inexpensive ECL solution which is an alternative to expensive commercial ECL solutions and which achieves a better signal and lower background than the commercial solutions tested.
\end{abstract}

(C) 2006 Elsevier B.V. All rights reserved.

Keywords: Enhanced chemiluminescence; Western blot; 4-iodophenylboronic acid; $p$-coumaric acid; 4-iodophenol

\section{Introduction}

The horseradish peroxidase (HRP)-catalysed enhanced chemiluminescence is widely used in basic

Abbreviations: 4IPBA, 4-iodophenylboronic acid; pCA, paracoumaric acid; 4IP, 4-iodophenol; ECL, enhanced chemiluminescence; HRP, horseradish peroxidase; Jak, Janus kinase; STAT, signal transducer and activator of transcription; BSA, bovine serum albumine; ICAM, intercellular adhesion molecule.

* Corresponding author. Tel.: +352 466644 6432; fax: +352 466644 6435.

E-mail address: claude.haan@uni.lu (C. Haan). research and has found its application in many commercial kits. One important application in life sciences is the ECL detection of Western blots. Proteins on Western blots are recognised by specific primary antibodies which are in turn detected by a secondary antibody coupled to HRP. HRP catalyzes the oxidation of luminol or other substrates in the presence of peroxidase and an enhancer. The light output is detected on film or by CCD camera-equipped documentation systems. The ECL detection has several advantages compared to colorimetric detection of Western blots. It is more sensitive and the blots can be reprobed with other primary antibodies after stripping of the membrane. 
A variety of different chemical compounds (e.g. coumaric acid, phenolic compounds, phenylboronic acid derivatives and others) can be used to enhance the light emission from the decay of the aminophtalate dianion, generated by the oxidation of luminol (Thorpe et al., 1985; Kricka et al., 1996b; Kuroda et al., 1999; Rehm, 2002). Different boronic acid derivatives were used as enhancer in the ECL detection of proteins on dot blots and Western blots with purified proteins but not with the aim of detecting endogenous amounts of proteins in Western blots of whole-cell lysates (Kricka et al., 1996a; Kuroda et al., 2001).

Here we compared alternative ECL solutions containing the different enhancers $p$-coumaric acid (pCA), 4-iodophenylboronic acid (4IPBA) or 4-iodophenol (4IP), to commercial ECL solutions. We were interested in obtaining a solution which would work well in detection of Western blots of total cell lysates, a situation in which the major problem is often not the detection limit but unspecific background chemiluminescence which can be due e.g. to unspecific primary and secondary antibody binding. The chemicals we used for the alternative ECL solutions are commercially available and inexpensive. We found that 4IPBA-ECL showed a very low background chemiluminescence allowing much longer exposure times than the other tested ECL solutions. We optimised the 4IPBA-ECL solution so that the signal intensity was superior to that of commercial kits and a previously described noncommercial ECL solution (Rehm, 2002).

\section{Materials and methods}

\subsection{The alternative, non-commercial ECL solutions}

DMSO stock solutions of $250 \mathrm{mM}$ luminol (Aldrich), $90 \mathrm{mM}$ para-coumaric acid (pCA) (Sigma), $90 \mathrm{mM} 4-$ iodophenylboronic acid (4IPBA) (Aldrich) and $90 \mathrm{mM} 4-$ iodophenol (4IP) (Aldrich) were prepared. The ECL solutions were generated by diluting luminol and one of the enhancers (pCA, 4IPBA or 4IP) in $100 \mathrm{mM}$ Tris/HCl $\mathrm{pH}$ 8.8. Hydrogenperoxyde (from a $30 \%$ stock solution) was added freshly to the ECL solutions just before detection of the membranes. The concentration of the enhancers (pCA, 4IPBA or 4IP), luminol and hydrogenperoxide were varied as described in the figure legends. The composition of the initial ECL solutions used to compare pCA and 4IPBA was as follows: $100 \mathrm{mM}$ Tris/ $\mathrm{HCl} \mathrm{pH} 8.8,2.5 \mathrm{mM}$ luminol, $0.4 \mathrm{mM}$ pCA or 4IPBA, $2.6 \mathrm{mM}$ hydrogenperoxide. The optimised 4IPBA-ECLsolution contains $100 \mathrm{mM}$ Tris/ $\mathrm{HCl} \mathrm{pH} 8.8,1.25 \mathrm{mM}$ luminol, $2 \mathrm{mM}$ 4IPBA, $5.3 \mathrm{mM}$ hydrogenperoxide.

\subsection{ECL detection in 96 well plates}

Each well contained a final volume of $100 \mu 1$ solution with a final concentration of $2.5 \mathrm{mM}$ luminol. The HRPcoupled antibody from Dako was used in a dilution of 1:1000. The concentration of the enhancers was varied as stated in the figure legend. To start the reaction, hydrogenperoxide was added to a final concentration of $2.6 \mathrm{mM}$. Chemiluminescence was observed using the CCD camera-equipped Chemi Lux detection system by Intas.

\subsection{Cell culture}

U4C and 2C4 cells (human fibrosarcoma cells kindly provided by Dr. I. M. Kerr, Cancer Research UK, London) were maintained in Dulbecco's modified Eagle's medium (Gibco) supplemented with $10 \%$ fetal calf serum, $100 \mathrm{mg} / 1$ streptomycin, and $60 \mathrm{mg} / 1$ penicillin. Cells were grown at $37{ }^{\circ} \mathrm{C}$ in a water-saturated atmosphere at $5 \% \mathrm{CO}_{2}$.

\subsection{Cell lysis and Western blot analysis}

All steps of cell lysis were performed at $4{ }^{\circ} \mathrm{C}$ using ice cold buffers. Cells were lysed on the dish with $500 \mu \mathrm{l}$ of lysis buffer containing 1\% Triton-X-100, $20 \mathrm{mM}$ Tris/ $\mathrm{HCl} \mathrm{pH}$ 7.5, $150 \mathrm{mM} \mathrm{NaCl}, 10 \mathrm{mM} \mathrm{NaF}, 1 \mathrm{mM}$ sodium vanadate, $10 \mathrm{mM}$ PMSF, $1 \mathrm{mM}$ benzamidine, $5 \mu \mathrm{g} / \mathrm{ml}$ aprotinin, $3 \mu \mathrm{g} / \mathrm{ml}$ pepstatin, $5 \mu \mathrm{g} / \mathrm{ml}$ leupeptin and $1 \mathrm{mM}$ EDTA. The cleared lysates were analyzed further by SDS-PAGE. The proteins were transferred to a polyvinylidene difluoride membrane (Amersham Pharmacia Biotech) and probed with the respective antibodies. Anti-Jak1 (610232, Transduction Laboratories), anti-calnexin (610523, Transduction Laboratories), antiSTAT-1 (610116, Transduction Laboratories), anti-actin (MAB1501, Chemicon) and anti-ICAM1 (G5, Santa Cruz Biotech.) were used for detection. The horseradish peroxidase-conjugated secondary goat-anti-mouse antibody was purchased from Dako (P0447). To ensure exactly the same treatment of the different blots during the Western blot detection procedure, multiple samples of the lysates and the molecular size marker were run on the same gel. After Western blotting, the uncut blot was treated with blocking solution (10\% BSA, $10 \mathrm{mM}$ Tris/ $\mathrm{HCl} \mathrm{pH} \mathrm{7.4,} 140 \mathrm{mM} \mathrm{NaCl}, 0.1 \% \mathrm{NP}-40)$ and then subsequently with the first and secondary antibody solutions (antibody diluted in $10 \mathrm{mM}$ Tris/ $\mathrm{HCl} \mathrm{pH} \mathrm{7.4,}$ $140 \mathrm{mM} \mathrm{NaCl}, 0.1 \% \mathrm{NP}-40$ ). The blot was cut just before detection with the different ECL solutions. Thus, only those blots were compared which had been exposed 
to identical electrophoresis, blotting, blocking and antibody-detection conditions. Having 22 slots on one gel, this limited the number of blots which could be compared to seven. The blots were always prepared freshly and were not stripped and reprobed to exclude effects due to previous detections. Blots were then incubated with the different ECL solutions. The "ECLWestern Blotting analysis system" (RPN2108, Amersham Biosciences), further named "commercial ECL-1" (Com1), and "SuperSignal West Pico chemiluminescent substrate" (\#34080, Pierce), further named "commercial ECL-2" (Com2), were used according to the manufacturers' protocols. The self made ECL solutions, containing pCA, 4IPBA or 4IP as described above, were incubated for $1 \mathrm{~min}$ with the blots before detection. The signals were detected either on film (Hyperfilm-ECL, Amersham Biosciences) or using the Chemi Lux ECL detection system (Intas). All the blots from one experiment (i.e. from one initial membrane from one SDS-PAGE gel) were detected at the same time with enough space in between them to prevent any mixing of different ECL solutions.

\section{Results and discussion}

\subsection{A 4IBPA-ECL solution combines high sensitivity and low background for Western blot detection}

The major challenge in protein detection on Western blots of total cell lysates is often not the detection limit but unspecific signals and background chemiluminescence. To distinguish the specific signal of a primary antibody from unspecific signals and the background we took advantage of a pair of well-characterized

A

WB: anti-Jak1

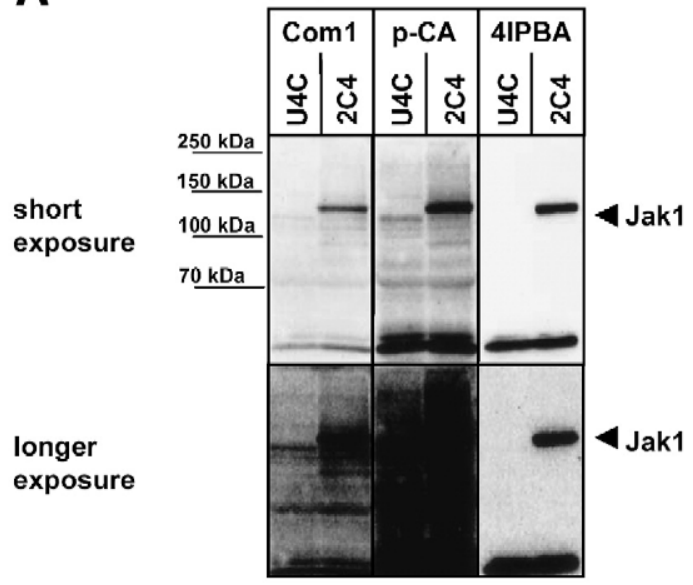

B

WB: anti-calnexin
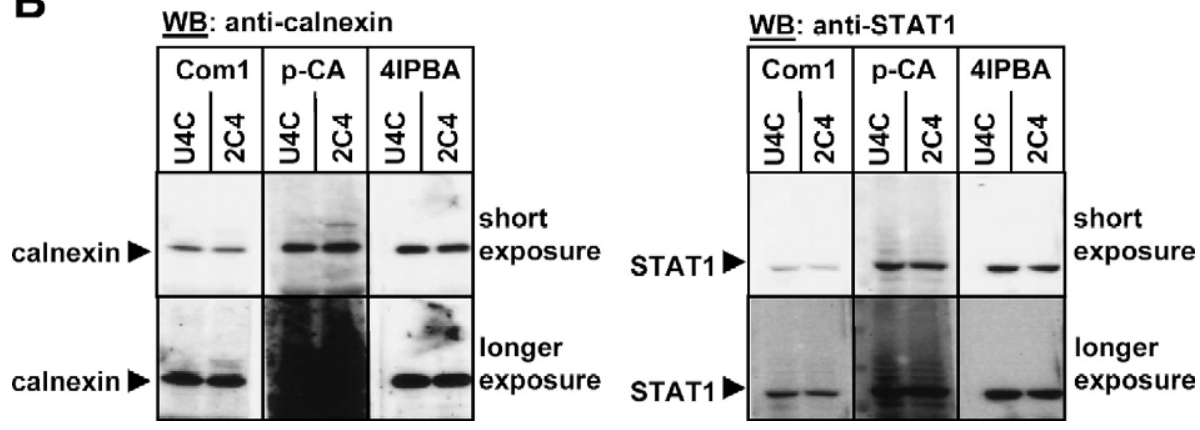

Fig. 1. The 4IBPA-ECL solution results in significantly less background chemiluminescence than the pCA-ECL solution. Lysates of U4C and 2C4 cells were separated by SDS-PAGE and transferred to PVDF membranes by electroblotting. The Western blots were detected using as primary antibodies (A) anti-Jak1 (610232, Transduction Laboratories) or (B) anti-calnexin (610523, Transduction Laboratories) and anti-STAT1 (610116, Transduction Laboratories). The blots were incubated with the various ECL solutions before detection with the Chemi Lux detection system from Intas. Detection times were chosen so that the signal strength and the different background chemiluminescence of the three solutions became apparent (short exposure: 1-2 $\mathrm{min}$; long exposure 5-10 min). The self made pCA- and 4IPBA-ECL solutions contained $2.5 \mathrm{mM}$ luminol, $2.6 \mathrm{mM}$ hydrogenperoxide and $0.4 \mathrm{mM}$ of either $p$-coumaric acid or 4-iodophenylboronic acid. The commercial ECL solution (Com1) was used according to the manufacturer's instructions. The position of molecular size markers is indicated in (A). 
fibrosarcoma cell lines expressing or lacking Janus kinase 1 (Jak1) (Kohlhuber et al., 1997; Behrmann et al., 2004). Jak 1 is a tyrosine kinase crucial for interferon signal transduction as well as for signalling mediated by cytokines of the IL-2 and IL-6 families (Yeh and Pellegrini, 1999; Heinrich et al., 2003). Western blots of lysates from 2C4 (Jak1-containing cells) and U4C cells (Jak1-deficient cells) were prepared as described in Materials and methods. In first experiments we used a non-commercial ECL solution based on coumaric acid as enhancer as published by Rehm (2002) (100 mM Tris/ $\mathrm{HCl} \mathrm{pH} 8.8,2.5 \mathrm{mM}$ luminol, $0.4 \mathrm{mM}$ pCA, $2.6 \mathrm{mM}$ hydrogenperoxide), and compared this to a solution with 4IPBA as enhancer, also at a $0.4 \mathrm{mM}$ concentration. Moreover, we included a commercially available ECL solution into our study.

Both non-commercial ECL solutions (pCA-ECL and 4IPBA-ECL) were superior to the tested commercial ECL solution "Com1" with regard to signal strength. The signal intensities of pCA-ECL- and 4IPBA-ECLsolutions were comparable in a detection with the Jak1 antibody (Fig. 1A). However, upon longer exposure the background observed in detections with the pCA-ECL solution was much higher than the one observed with the 4IPBA-ECL solution (Fig. 1A, lower panel). This allowed for much longer exposure times when using the 4IPBA solution. Also the "Com1" solution showed a higher background than the 4IPBA-ECL solution (Fig. 1A, lower panel). Also for other primary antibodies (anti-calnexin, anti-STAT1) the signal intensity of the non-commercial ECL solutions was higher than for the commercial solution (Fig. 1B).

\subsection{The non-commercial ECL solutions show a fast onset of chemiluminescence and a robust signal over time}

A useful Western blot ECL detection reagent has several other characteristics besides showing a good signal intensity. The onset of the signal should be rapid. The duration of the signal should be as long as possible to achieve a good signal accumulation over time and to offer a sufficiently broad time window for successive exposures. We measured the chemiluminescence with a CCD camera-based system for identical intervals of time, starting at different time points after addition of the ECL reagents to the Western blots ( $1 \mathrm{~min}$ to $30 \mathrm{~min}$ ). Both noncommercial ECL solutions (pCA-ECL and 4IPBA-ECL) showed a quick onset of chemiluminescence $(t=1 \mathrm{~min}$, Fig. 2A) and the signal was sustained for $30 \mathrm{~min}$ minimum $(t=30 \mathrm{~min}$, Fig. 2A). For the whole time period the background chemiluminescence was higher for the $\mathrm{pCA}-$ ECL solution than for the 4IBPA-ECL solution.
3.3. A solution assay is not suited to optimize ECL conditions for Western blots

To rapidly test concentration dependencies of the various ECL solution components and other additives
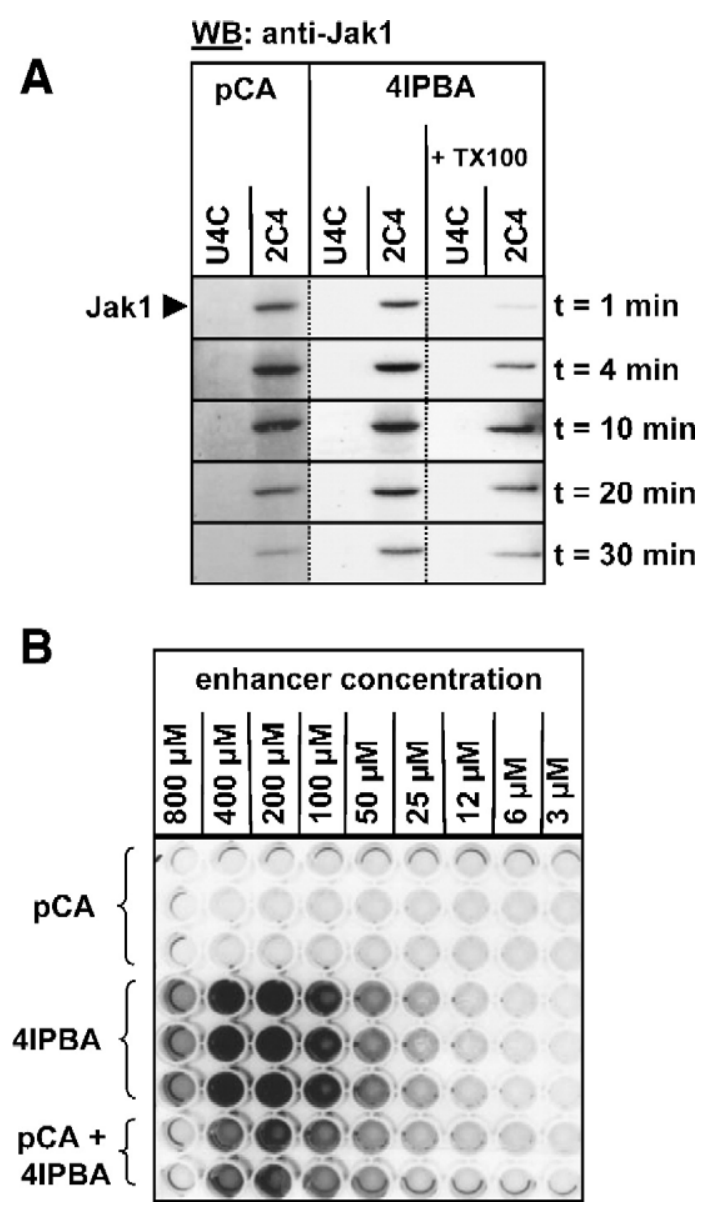

Fig. 2. ECL solutions generate different effects in the Western blot detection than in the solution assay. (A) Lysates of U4C and 2C4 cells were separated by SDS-PAGE and transferred to PVDF membranes by electroblotting. The Western blots were then detected using an antiJak1 primary antibody (610232, Transduction Laboratories). The blots were incubated for $1 \mathrm{~min}$ with the various ECL solutions and then detected with the Chemi Lux detection system from Intas for identical intervals ( $1 \mathrm{~min})$, starting at different time points after the beginning of the detection, as indicated. The 4IPBA- and the pCA-ECL solutions were composed as described in the legend of Fig. 1. Triton X-100 (TX100) was added to the 4IPBA-ECL solution to a percentage of $0.25 \%$. (B) The concentration dependancy of pCA and 4IPBA on chemiluminescence was investigated in a 96 well assay as described in Materials and methods. Each well contained a final concentration of $2.5 \mathrm{mM}$ luminol and HRP-conjugated secondary antibody in a dilution of 1:1000. The concentrations of the enhancers p-CA and 4-IPBA were varied from $800 \mu \mathrm{M}$ to $3 \mu \mathrm{M}$. To start the reaction, hydrogenperoxide was added to a final dilution of $2.6 \mathrm{mM}$. Chemiluminescence was observed using the Chemi Lux detection system from Intas. 
on chemiluminescence, we intended to use a 96-well plate solution assay (see Materials and methods).

Both enhancers showed an important enhancement over the non-enhanced reaction (data not shown). Upon variation of the enhancer concentrations between $800 \mu \mathrm{M}$ and $3 \mu \mathrm{M}$ the chemiluminescence was much greater for 4IPBA than for pCA (Fig. 2B) in the 96 well solution assay. $400 \mu \mathrm{M}$ was the optimal concentration. There were no additive or synergistic effects when both enhancers were combined; in fact, the chemiluminescence observed was lower than that observed for 4IPBA alone (Fig. 2B). However, the strikingly better performance of 4IPBA

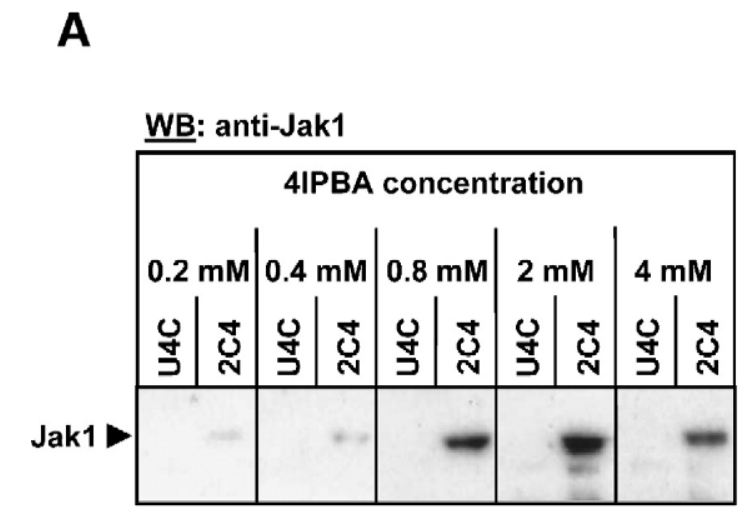

$\mathbf{B}$

BB: anti-Jak1
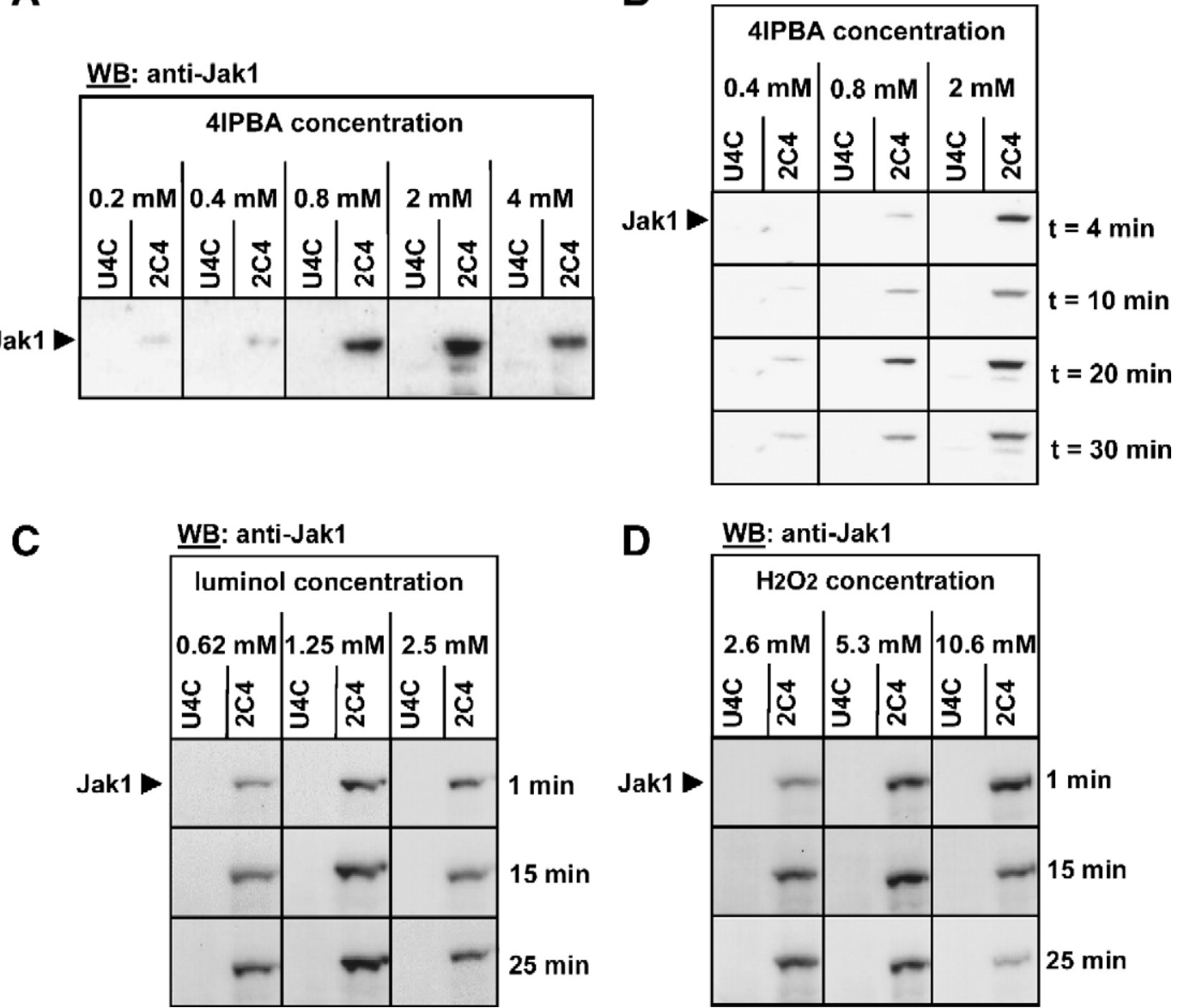

observed in the solution assay is in contrast to the Western blot results where pCA and 4IPBA displayed a comparable sensitivity (Figs. 1 and 2A).

We also investigated the effects of a number of additives. Detergents were described to have positive effects on the increase of specific light emissions (Iwata et al., 1995). Different detergents were tested in the 96 well assay. Triton $\mathrm{X}-100$ at concentrations between $0.25 \%$ and $0.5 \%$ prolonged chemiluminescence in the solution assay (data not shown) but it delayed the chemiluminescence in the Western blot detection and shortened it at later times (Fig. 2A).

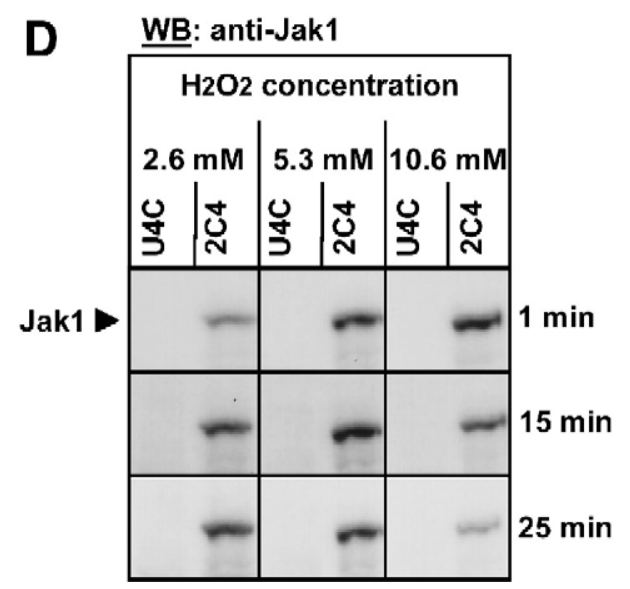

Fig. 3. Optimisation of the 4IPBA-ECL solution for Western blot detection. Identical Western blots of lysates from 2C4 and U4C cells were detected with a Jak1-specific primary antibody, as described in the legend of Fig. 1. The concentration of the constituents of the 4IPBA-ECL solution was varied in an attempt to optimise the chemiluminescent signal. Chemiluminescence was observed using the Chemi Lux detection system from Intas. (A) The concentration of 4IPBA was varied between 0.2 and $4 \mathrm{mM}$. The concentrations of the other components of the ECL solution were unchanged (100 mM Tris/HCl pH 8.8, $2.5 \mathrm{mM}$ luminol, $2.6 \mathrm{mM}$ hydrogenperoxide). The signal was detected for $1 \mathrm{~min}$. (B) Blots were treated as described in (A). The signal was detected for identical intervals $(1 \mathrm{~min})$ starting at various time points after the beginning of the detection, as indicated. (C) Various concentrations of luminol $(0.62 \mathrm{mM}, 1.25 \mathrm{mM}, 2.5 \mathrm{mM})$ were tested while the other components were $2 \mathrm{mM} 4 \mathrm{IPBA}$ and $2.6 \mathrm{mM}$ hydrogenperoxide. The signal was detected for identical intervals $(1 \mathrm{~min})$ starting at various time points after the beginning of the detection, as indicated. (D) Hydrogenperoxide concentrations of $2.6 \mathrm{mM}, 5.3 \mathrm{mM}$ and $10.6 \mathrm{mM}$ were tested. The solutions also contained $2 \mathrm{mM} 4 \mathrm{IPBA}, 2.5 \mathrm{mM}$ luminol. The signal was detected for identical intervals $(1 \mathrm{~min})$ starting at various time points after the beginning of the detection, as indicated. 
Unfortunately, other additives which were described to enhance chemiluminescence in solution assays like ammoniumpersulfate and $m$-chloroperbenzoic acid (Motsenbocker and Kondo, 1994; Yeh and Lin, 2002) rather worsened Western blot results (data not shown). Thus, obviously because of the different assay conditions, we could not use the convenient and fast solution assay for the optimization of the ECL solution in Western blot detections. Another group encountered comparable problems and reported that polymers stabilized the HRP-mediated light emission in solution but not in a dot blot or microwell immunoassay (Ji and Kricka, 1996).

\subsection{Optimisation of the 4IPBA-ECL solution for Western blot detection}

In an attempt to optimise the solution, we investigated the effect of various 4IPBA concentrations (0.2 mM, $0.4 \mathrm{mM}, 0.8 \mathrm{mM}, 2 \mathrm{mM}, 4 \mathrm{mM})$ on signal intensity. The concentrations of the other components of the ECL solution were unchanged $(100 \mathrm{mM}$ Tris/HCl pH 8.8, $2.5 \mathrm{mM}$ luminol, $2.6 \mathrm{mM}$ hydrogenperoxide). As shown in Fig. 3A, the strongest signal was achieved at a concentration of $2 \mathrm{mM}$ 4IPBA. The signal was stable over time and could be detected readily also 30 min after the start of the detection (Fig. 3B).

When we tested a number of luminol concentrations $(0.62 \mathrm{mM}, 1.25 \mathrm{mM}, 2.5 \mathrm{mM})$ at a 4IPBA concentration of $2 \mathrm{mM}$, we found that a concentration of $1.25 \mathrm{mM}$ generated the strongest signal over time (Fig. 3C).

Finally, we investigated the effect of hydrogenperoxide concentrations on signal intensity. It was reported previously that HRP is inhibited by concentrations of hydrogenperoxide above $4.4 \mathrm{mM}$ (Ilyina et al., 2000). On the other hand, it was described that 4-iodophenylboronic acid reacts with hydrogenperoxide to boric acid and 4-iodophenol (Handley et al., 2004), another well known enhancer of chemiluminescent reactions (Thorpe et al., 1985). This happens within minutes after the addition of hydrogenperoxide. Thus, 4-iodophenol might be the actual enhancer in this system and not 4-iodophenylboronic acid. Testing different concentrations of hydrogenperoxide (2.6 mM, 5.3 mM, $10.6 \mathrm{mM})$ at a 4IPBA concentration of $2 \mathrm{mM}$, we found that the chemiluminescence signal intensity was enhanced when we increased the concentration to $5.3 \mathrm{mM}$ and that the signal was stronger over time if compared to higher or lower hydrogenperoxide concentrations. Higher concentrations led to a decrease of chemiluminescence with time while for lower concentrations the chemiluminescent signal was delayed (Fig. 3D).
3.5. Comparison of 4IBPA and 4-iodophenol (4IP) as enhancers for Western blot detection

Because 4IPBA was described to quantitatively react to 4-iodophenol (4IP) and boric acid in the presence of hydrogenperoxide (Handley et al., 2004) we now included 4-iodophenol (4IP) into our study. We analysed if the 4IP- and the 4IPBA-ECL behaved identically. Testing different concentrations of 4IP in the ECL solution showed that 4IP was in fact a stronger enhancer than 4IPBA. At a concentration of $0.2 \mathrm{mM}$, 4IP led to a signal of comparable intensity as seen for $0.4 \mathrm{mM}$ 4IPBA (Fig. 4A). However, the background signal was also elevated. Fig. 4B shows that the signal intensity observed when using an ECL solution containing $2 \mathrm{mM}$ of 4IPBA is comparable to the one resulting

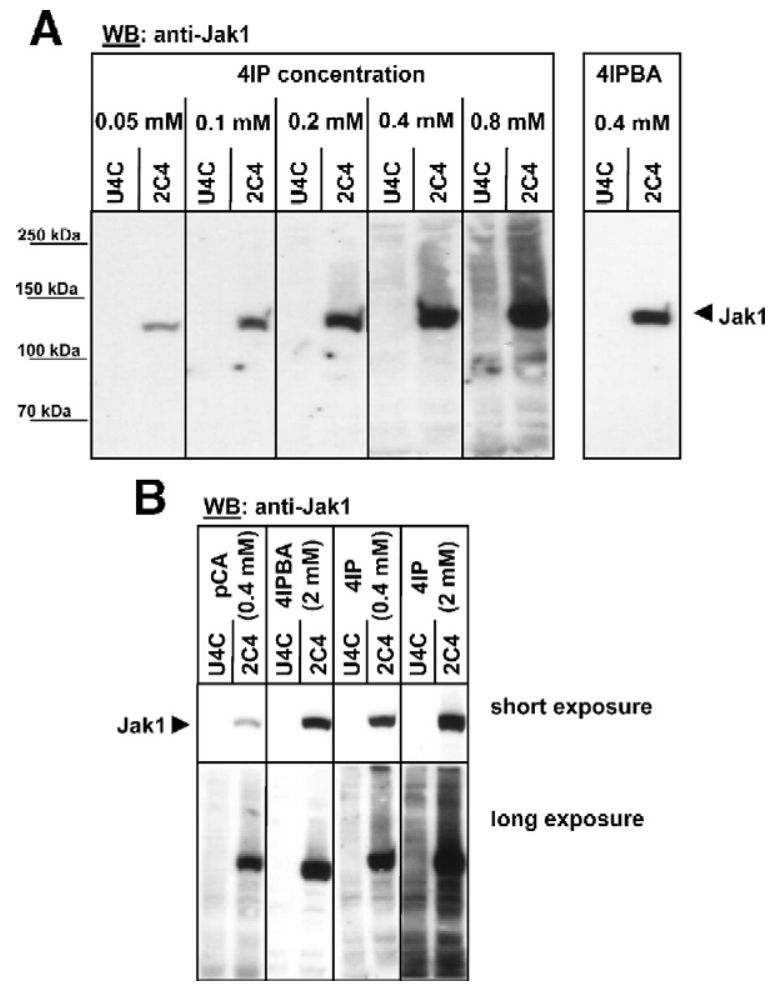

Fig. 4. Comparison of 4IBPA and 4-iodophenol (4IP) as enhancers for Western blot detection. (A) Identical Western blots of lysates from 2C4 and U4C cells were detected with ECL solutions containing $100 \mathrm{mM}$ Tris/ $\mathrm{HCl} \mathrm{pH} 8.8,2.5 \mathrm{mM}$ luminol, $2.6 \mathrm{mM}$ hydrogenperoxide and either $0.4 \mathrm{mM} 4 \mathrm{IPBA}$ or varying concentrations of 4IP $(0.05 \mathrm{mM}-$ $0.8 \mathrm{mM}$ ). Chemiluminescence was measured for a two minute interval using the Chemi Lux detection system from Intas. The position of molecular size markers is indicated. (B) Western blots were detected with ECL solutions containing $100 \mathrm{mM}$ Tris/ $\mathrm{HCl} \mathrm{pH} 8.8,2.5 \mathrm{mM}$ luminol, $2.6 \mathrm{mM}$ hydrogenperoxide and either $0.4 \mathrm{mM} \mathrm{pCA}, 2 \mathrm{mM}$ 4IPBA, $0.4 \mathrm{mM}$ 4IP or $2 \mathrm{mM}$ 4IP. Chemiluminescence was measured for a two minute interval using the Chemi Lux detection system from Intas. 
from a $0.4 \mathrm{mM}$ 4IP solution. However, because of the higher background chemiluminescence, using 4IP instead of 4IPBA does not offer an advantage.

\subsection{The optimised 4IPBA-ECL solution shows higher signal intensity than commercial ECL solutions and has a lower background than $p C A-E C L$ and $4 I P-E C L$}

It remained to be tested if a 4IPBA solution containing all constituents in the optimal concentrations as determined from the individual optimisations would lead to a superior ECL solution. Western blots of lysates from $2 \mathrm{C} 4$ and $\mathrm{U} 4 \mathrm{C}$ cells were detected with two commercial (Com1 and Com2) and two alternative ECL solutions (pCA and 4IP) as well as with the now optimised 4IPBA-ECL solution. The pCA-ECL and 4IP-ECL solution contained $100 \mathrm{mM}$ Tris/ $\mathrm{HCl} \mathrm{pH} 8.8$, $2.5 \mathrm{mM}$ luminol, $2.6 \mathrm{mM}$ hydrogenperoxide and $0.4 \mathrm{mM}$ pCA or 4IP. The optimised 4IPBA-ECL solution contained $100 \mathrm{mM}$ Tris/ $\mathrm{HCl} \mathrm{pH} 8.8,1.25 \mathrm{mM}$ luminol, $5.3 \mathrm{mM}$ hydrogenperoxide and $2 \mathrm{mM}$ 4IPBA. In the Western blot detection with the Jak1 primary antibody, the latter solution generated a significantly stronger specific signal than the other ECL solutions (Fig. 5A). The signal also remained the most intense one over time (data not shown). Upon longer exposure, the background chemiluminescence was weak compared to the other non-commercial ECL solutions (Fig. 5A). The

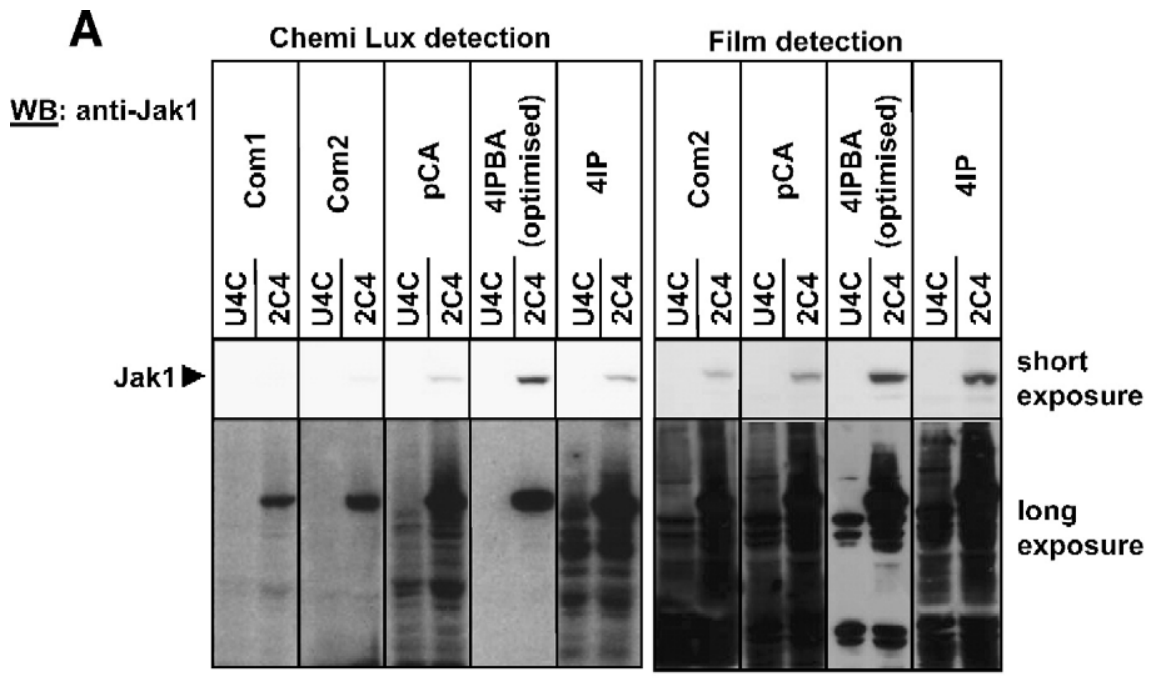

B

WB: anti-actin

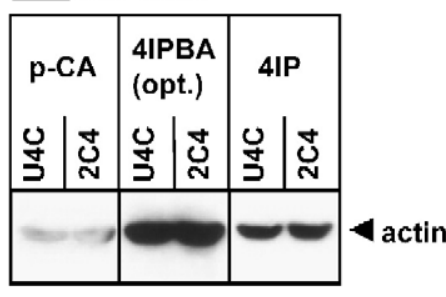

WB: anti-ICAM1

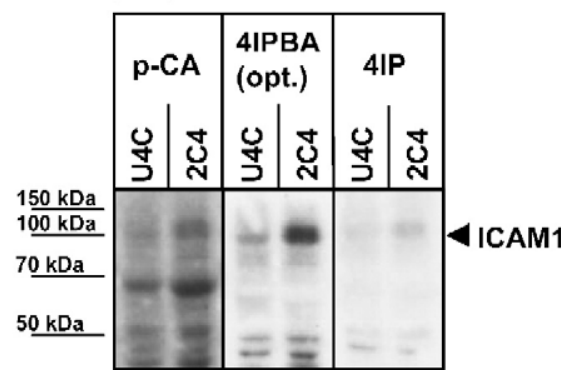

Fig. 5. The optimised 4IPBA-ECL solution shows higher signal intensity than commercial ECL solutions and has a lower background than pCAECL and 4IP-ECL. (A) Identical Western blots of lysates from 2C4 and U4C cells were detected with a primary antibody against Jak1. Enhanced chemiluminescence was observed after incubation with a number of ECL solutions. Commercial ECL solutions Com1 and Com2 were used according to the manufacturers' protocols. The self made pCA- and 4IP-ECL solutions contained $100 \mathrm{mM} \mathrm{Tris/HCl} \mathrm{pH} 8.8,2.5 \mathrm{mM}$ luminol, $2.6 \mathrm{mM}$ hydrogenperoxide and either $0.4 \mathrm{mM} \mathrm{pCA}$ or $0.4 \mathrm{mM}$ 4IP. The composition of the optimised 4IPBA-ECL was as follows: $100 \mathrm{mM}$ Tris/HCl $\mathrm{pH}$ 8.8, $1.25 \mathrm{mM}$ luminol, $5.3 \mathrm{mM}$ hydrogenperoxide and $2 \mathrm{mM}$ 4IPBA. Chemiluminescence was observed for one experiment using the Chemi Lux detection system from Intas or for a different series of Western blots by film detection (Hyperfilm-ECL, Amersham Biosciences). Detection times were chosen so that signal strength and the different background chemiluminescence of the solutions became apparent (short exposure Chemi Lux: $2 \mathrm{~min}$; long exposure Chemi Lux: $10 \mathrm{~min}$; short exposure film: $15 \mathrm{~s}$; long exposure film: $5 \mathrm{~min}$ ). (B) Western blots were detected with anti-actin (MAB1501, Chemicon) or anti-ICAM1 (G5, Santa Cruz Biotech.) antibodies. ECL detection was performed with the same non-commercial ECL solutions described in (A). Exposure times were $1 \mathrm{~min}$ for the anti-actin- and $2 \mathrm{~min}$ for the anti-ICAM-detection. 
background for 4IPBA-ECL was also lowest when the ECL signals were detected on film (Fig. 5A).

Also when using other primary antibodies, e.g. antiactin or anti-ICAM1, the optimised 4IPBA solution generated very strong signals compared to the other noncommercial ECL solutions (Fig. 5B). Interestingly, we observed that ICAM1 levels are reduced in U4C cells that do not express Jak1. As inducible ICAM-1 expression is known to depend on Jak/STAT signal transduction, e.g. after IFN $\gamma$-stimulation (Walter et al., 1997), it is conceivable that low-level Jak/STAT signalling (possibly by an autocrine stimulation) may also crucially contribute to the constitutive expression of ICAM-1. This will be addressed in further studies.

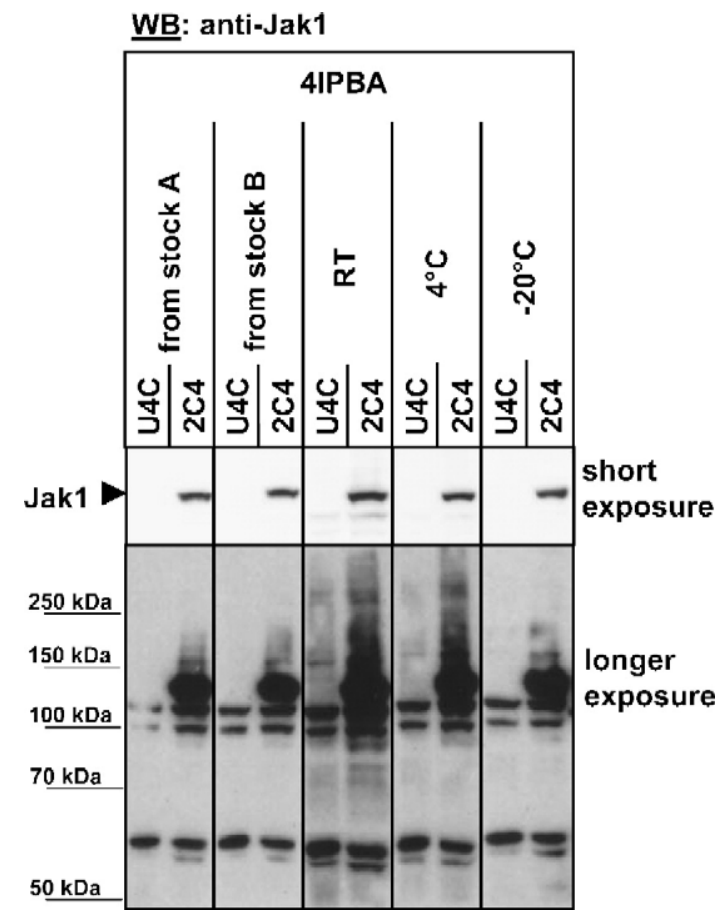

Fig. 6. The 4IPBA solution is stable over time when stored at $4{ }^{\circ} \mathrm{C}$. Identical Western blots of lysates from $2 \mathrm{C} 4$ and $\mathrm{U} 4 \mathrm{C}$ cells were detected with a primary antibody against Jak1. Enhanced chemiluminescence was observed after incubation with a number of ECL solutions. The composition of all the final solutions was identical (100 mM Tris/HCl pH 8.8, $1.25 \mathrm{mM}$ luminol, $5.3 \mathrm{mM}$ hydrogenperoxide and $2 \mathrm{mM} 4 \mathrm{IPBA})$. One solution has been stored either at room temperature (RT), at $4{ }^{\circ} \mathrm{C}$ or at $-20{ }^{\circ} \mathrm{C}$ for one month. The solutions were stored without addition of $5.3 \mathrm{mM}$ hydrogenperoxide which was added fresh just before detection. Two other solutions were freshly prepared from stocks. One was prepared from fresh stocks (Stock A). The other was prepared from stock solutions stored at room temperature for 1 month (Stock B). Chemiluminescence was observed using the Chemi Lux detection system from Intas. Detection times were chosen so that the signal strength and the different background chemiluminescence of the three solutions became apparent (short exposure: $1 \mathrm{~min}$; long exposure: $5 \mathrm{~min}$ ).
Moreover, we have also optimised the pCA protocol (pCA optimised: $100 \mathrm{mM}$ Tris/ $\mathrm{HCl} \mathrm{pH} 8.8,2.5 \mathrm{mM}$ luminol, $2.6 \mathrm{mM}$ hydrogenperoxide and $0.2 \mathrm{mM} \mathrm{pCA}$ ). This optimised pCA-ECL has a comparable signal intensity to the optimised 4IPBA-ECL but still shows higher background chemiluminescence (data not shown).

\subsection{IPBA solution is stable over time when stored at $4{ }^{\circ} \mathrm{C}$}

The stability of the solutions over time was tested by leaving the 4IPBA-ECL-solution at room temperature (RT), at $4{ }^{\circ} \mathrm{C}$ or at $-20{ }^{\circ} \mathrm{C}$ for one month. These solutions were tested in Western blot detections in comparison to solutions that were freshly prepared from stocks: either from freshly made ones (stock A) or from stocks which had been prepared one month ago and stored at room temperature (stock B). All the solutions generated signals of comparable strength in the Western blot detection (Fig. 6). We conclude that the solution is stable under all conditions tested for one month minimum. The background chemiluminescence, however, was highest in the solution stored for one month at room temperature followed by the solution stored at $4{ }^{\circ} \mathrm{C}$ (Fig. 6, lower panel). Thus, for optimal results we recommend the fresh preparation of the solution from stocks.

\section{Concluding remarks}

We have presented an effective, optimised protocol for Western blot detection that offers an excellent performance at low cost. In a comparison of different non-commercial ECL-solutions with commercial solutions we have demonstrated the advantages of the 4IPBA-ECL-solution, which combines better sensitivity and low background chemiluminescence. This ECL solution is useful in Western blot detections of total cell lysates, where background chemiluminescence is often high. Of note, the pCA- and 4IPBA-ECL-solutions can, after the addition of hydrogenperoxide, be used several times to detect Western blots during the same day but should not be used longer (just as the commercial solutions). The alternative ECL solutions do not contain chemicals which harm the blots: We regularly redetect Western blots after stripping for up to five times with these reagents (data not shown). Due to the strong signals and the low background of the 4IPBA-ECL solution, the primary and secondary antibodies used for detection can also be used at lower concentrations. This again reduces the cost and may 
help to save resources in the case of limited stocks of antibody. Furthermore, "self made" ECL solutions allow maximum flexibility: For a given antibody one can easily optimise conditions if this is necessary. The novel protocol is of special interest in applications where lowest possible background is needed.

\section{Acknowledgement}

We are grateful to Dr. I. M. Kerr (Cancer Research $\mathrm{UK}$, London) for providing $2 \mathrm{C} 4$ and $\mathrm{U} 4 \mathrm{C}$ cells. This work has been supported by a grant from the University of Luxembourg, project R1F105L01.

\section{References}

Behrmann, I., Smyczek, T., Heinrich, P.C., Schmitz-Van de Leur, H., Komyod, W., Giese, B., Müller-Newen, G., Haan, S., Haan, C., 2004. Janus kinase (Jak) subcellular localization revisited: the exclusive membrane localization of endogenous Janus kinase 1 by cytokine receptor interaction uncovers the Jak.receptor complex to be equivalent to a receptor tyrosine kinase. J. Biol. Chem. 279, 35486.

Handley, R.S., Eickholt, R.A., Schaap, A.P., Akhavan-Tafti, H., 2004. On the mechanism of arylboronic acid "enhancement" of peroxidasecatalyzed reactions. Abstract of the 13th International Symposium on Bioluminescence \& Chemiluminescence. Luminescence 19, 144.

Heinrich, P.C., Behrmann, I., Haan, S., Hermanns, H.M., MüllerNewen, G., Schaper, F., 2003. Principles of interleukin (IL)-6-type cytokine signalling and its regulation. Biochem. J. 374, 1.

Ilyina, A.D., Mauricio, B.J.E., Sifuentes, S.I.P., Martinez, H.J.L., Bogatcheva, E.S., Romero, G.J., Rodriguez, M.J., 2000. Behavior of enhanced chemiluminescence peroxidase-catalyzed peroxidation of luminol in the system of surfactant-water-organic solvent. Mosc. Univ. Chem. Bull. 41, 109.

Iwata, R., Ito, H., Hayashi, T., Sekine, Y., Koyama, N., Yamaki, M., 1995. Stable and general-purpose chemiluminescent detection system for horseradish peroxidase employing a thiazole compound enhancer and some additives. Anal. Biochem. 231, 170.

Ji, X., Kricka, L.J., 1996. Effect of polymers on enhanced chemiluminescent assays for peroxidase and peroxidase labels. J. Biolumin. Chemilumin. 11, 303.
Kohlhuber, F., Rogers, N.C., Watling, D., Feng, J., Guschin, D., Briscoe, J., Witthuhn, B.A., Kotenko, S.V., Pestka, S., Stark, G.R., Ihle, J.N., Kerr, I.M., 1997. A JAK1/JAK2 chimera can sustain alpha and gamma interferon responses. Mol. Cell. Biol. 17, 695.

Kricka, L.J., Cooper, M., Ji, X., 1996a. Synthesis and characterization of 4-iodophenylboronic acid: a new enhancer for the horseradish peroxidase-catalyzed chemiluminescent oxidation of luminol. Anal. Biochem. 240, 119.

Kricka, L.J., Ji, X., Thorpe, G.H., Edwards, B., Voyta, J., Bronstein, I., 1996b. Comparison of 5-hydroxy-2, 3-dihydrophthalazine-1, 4dione and luminol as co-substrates for detection of horseradish peroxidase in enhanced chemiluminescent reactions. J. Immunoass. $17,67$.

Kuroda, N., Kawazoe, K., Nakano, H., Wada, M., Nakashima, K., 1999. New phenylboronic acid derivatives as enhancers of the luminol- $\mathrm{H}(2) \mathrm{O}(2)$-horseradish peroxidase chemiluminescence reaction. Luminescence 14, 361.

Kuroda, N., Murasaki, N., Wada, M., Nakashima, K., 2001. Application of an enhanced luminol chemiluminescence reaction using 4-[4,5-di(2-pyridyl)-1H-imidazol-2-yl]phenylboronic acid to photographic detection of horseradish peroxidase on a membrane. Luminescence 16, 167.

Motsenbocker, M.A., Kondo, K., 1994. Improvements to enhanced horseradish peroxidase detection sensitivity. J. Biolumin. Chemilumin. 9, 15.

Rehm, H., 2002. Der Experimentator: Proteinbiochemie/Proteomics. Spektrum Akad. Verlag, Heidelberg.

Thorpe, G.H., Kricka, L.J., Moseley, S.B., Whitehead, T.P., 1985. Phenols as enhancers of the chemiluminescent horseradish peroxidase-luminol-hydrogen peroxide reaction: application in luminescence-monitored enzyme immunoassays. Clin. Chem. 31, 1335.

Walter, M.J., Look, D.C., Tidwell, R.M., Roswit, W.T., Holtzman, M.J., 1997. Targeted inhibition of interferon-gamma-dependent intercellular adhesion molecule-1 (ICAM-1) expression using dominantnegative Stat1. J. Biol. Chem. 272, 28582.

Yeh, H.C., Lin, W.Y., 2002. Enhanced chemiluminescence for the oxidation of luminol with m-chloroperoxybenzoic acid catalyzed by microperoxidase 8. Anal. Bioanal. Chem. 372, 525.

Yeh, T.C., Pellegrini, S., 1999. The Janus kinase family of protein tyrosine kinases and their role in signaling. Cell. Mol. Life Sci. 55, 1523. 